\title{
Pharmacokinetic, Hemostatic, and Anticancer Properties of a Low-Anticoagulant Bovine Heparin
}

\author{
Roberto P. Santos ${ }^{1, *}$ Ana M.F. Tovar ${ }^{1, *}$ Marcos R. Oliveira ${ }^{1}$ Adriana A. Piquet ${ }^{1}$ Nina V. Capillé \\ Stephan N.M.C.G. Oliveira ${ }^{1}$ Ana H. Correia ${ }^{2}$ José N. Farias ${ }^{3}$ Eduardo Vilanova $^{1}$ Paulo A.S. Mourão ${ }^{1}$
}

${ }^{1}$ Hospital Universitário Clementino Fraga Filho and Instituto de Bioquímica Médica Leopoldo de Meis, Laboratório de Tecido Conjuntivo, Hospital Universitário Clementino Fraga Filho and Instituto de Bioquímica Médica Leopoldo de Meis, Universidade Federal do Rio de Janeiro, Rio de Janeiro, Brazil

${ }^{2}$ Hospital Universitário Clementino Fraga Filho, Serviço de Anatomia Patológica, Hospital Universitário Clementino Fraga Filho, Rio de Janeiro, Brazil

${ }^{3}$ Hospital Universitário Clementino Fraga Filho, Laboratório Multidisciplinar de Pesquisa, Hospital Universitário Clementino Fraga Filho, Rio de Janeiro, Brazil
Address for correspondence Paulo A.S. Mourão, PhD, Hospital Universitário Clementino Fraga Filho, Rua Professor Rodolpho Paulo Rocco 255, sala 4A01, 21941-913 Rio de Janeiro, Brazil (e-mail: pmourao@hucff.ufrj.br).

TH Open 2022;6:e114-e123.

\begin{abstract}
Keywords

- unfractionated heparin

- low-molecular weight heparin

- pharmacokinetics

- bleeding

- Lewis lung carcinoma

Heparin is a centennial anticoagulant drug broadly employed for treatment and prophylaxis of thromboembolic conditions. Although unfractionated heparin (UFH) has already been shown to have remarkable pharmacological potential for treating a variety of diseases unrelated with thromboembolism, including cancer, atherosclerosis, inflammation, and virus infections, its high anticoagulant potency makes the doses necessary to exert nonhemostatic effects unsafe due to an elevated bleeding risk. Our group recently developed a new low-anticoagulant bovine heparin $(\mathrm{LABH})$ bearing the same disaccharide building blocks of the UFH gold standard sourced from porcine mucosa (HPI) but with anticoagulant potency approximately $85 \%$ lower (approximately 25 and 180 Heparin International Units $[\mathrm{IU}] / \mathrm{mg})$. In the present work, we investigated the pharmacokinetics profile, bleeding potential, and anticancer properties of LABH administered subcutaneous into mice. LABH showed pharmacokinetics profile similar to HPI but different from the low-molecular weight heparin (LMWH) enoxaparin and diminished bleeding potential, even at high doses. Subcutaneous treatment with LABH delays the early progression of Lewis lung carcinoma, improves survival, and brings beneficial health outcomes to the mice, without the advent of adverse effects (hemorrhage/mortality) seen in the animals treated with HPI. These results demonstrate that $\mathrm{LABH}$ is a promising candidate for prospecting new therapeutic uses for UFH.
\end{abstract}

\section{Introduction}

Unfractionated heparin (UFH) obtained from animal tissues has been massively exploited as anticoagulant agent for

\footnotetext{
These authors contributed equally as first authors.
}

received

September 13, 2021

accepted after revision January 20, 2022
DOI https://doi.org/

10.1055/a-1750-1300.

ISSN 2512-9465. almost a century, which makes it one of the oldest extant biologic drugs. ${ }^{1}$ Although low-molecular weight heparins (LMWHs) and directly acting oral anticoagulants have been increasingly prescribed for treatment and prophylaxis of most thromboembolic diseases, UFH is still the most potent anticoagulant, being indispensable for patients

(c) 2022. The Author(s).

This is an open access article published by Thieme under the terms of the Creative Commons Attribution License, permitting unrestricted use, distribution, and reproduction so long as the original work is properly cited. (https://creativecommons.org/licenses/by/4.0/)

Georg Thieme Verlag KG, Rüdigerstraße 14, 70469 Stuttgart, Germany 
requiring a rapid-onset and deep low-coagulant state, such as those undergoing cardiopulmonary bypass, extracorporeal membrane oxygenation, hemodialysis, and severe deep-vein thrombosis and pulmonary embolism. ${ }^{2}$ In addition to anticoagulant activity, UFH also exerts many other biological effects, including modulation of different proteases and components of the extracellular matrix and binding to cytokines and growth factors. ${ }^{3}$

Several preclinical and clinical studies have already demonstrated that UFH has a remarkable pharmacological potential for treating diseases unrelated to thromboembolism such as cancer, atherosclerosis, inflammation, and viral infections. ${ }^{4,5}$ Among them, we can highlight the therapeutic effects of UFH on different pathways related to cancer progression, including angiogenesis, tumor cell proliferation and adhesion, immune system modulation and tumor cell migration and invasion during metastasis. ${ }^{6}$

However, the high anticoagulant potency of UFH makes the doses commonly necessary to achieve satisfactory effects on therapeutic targets related to cancer or other non-thromboembolic diseases unsafe due to an elevated risk of hemorrhage incidents. ${ }^{7}$ In addition to this serious adverse effect, UFH is clinically employed by intravenous route, making it unfeasible for long-term outpatient treatments. ${ }^{8}$ Although LMWHs and some UFH mimetics obtained through extensive chemical/enzymatic processes have already proven to be effective and pose reduced risk of bleeding, different stakeholders involved in the production and research of heparins are still looking for new and more feasible UFH derivatives that somehow preserve the physical-chemical features required for aiming new therapeutic targets but with decreased anticoagulant activity nonetheless. ${ }^{9}$

All UFHs currently available for clinical use and production of LMWHs are produced using heparin porcine intestine (Heparin Porcine Intestine; HPI) (Heparin Bovine Intestine; HBI) Hepatocyte Growth Factor (HGF) as raw material, except in some countries, including Brazil, Argentina, and India, which also employ UFH products obtained from heparin bovine intestine (HBI). ${ }^{10-13}$ However, the use of HPI and $\mathrm{HBI}$ as interchangeable UFHs requires special attention due to their chemical and pharmacological differences. ${ }^{14}$ The
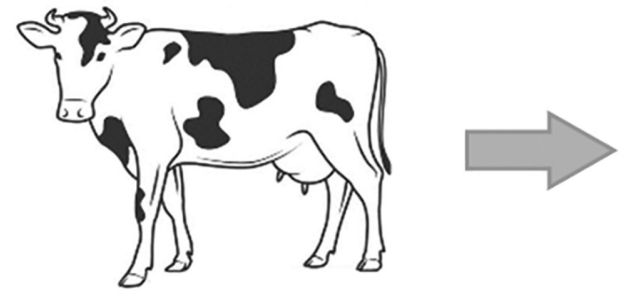

\section{Pharmaceutical HBI $(\sim 100 \mathrm{lU} / \mathrm{mg})$}
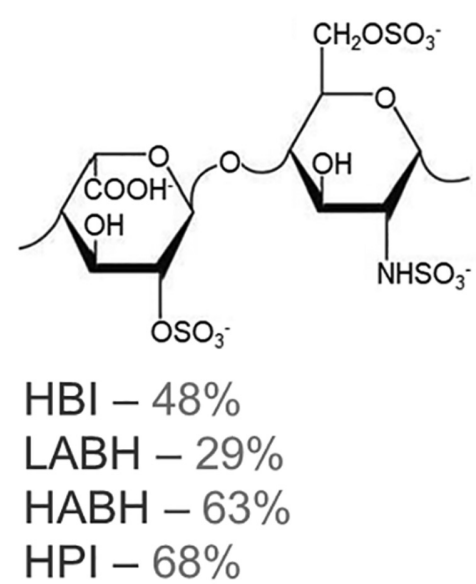

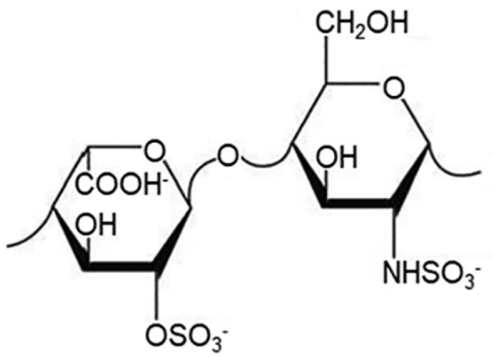

$\mathrm{HBI}-28 \%$

$$
\begin{aligned}
& \text { LABH }-50 \% \\
& \text { HABH - 8\% } \\
& \text { HPI }-3 \%
\end{aligned}
$$

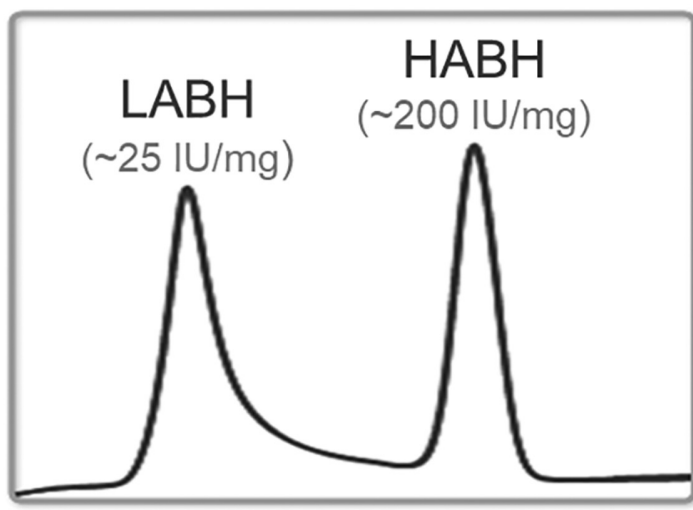

HBl ion-exchange chromatography

Fig. 1 Novel bovine heparins. Average pharmaceutical bovine heparin preparations (HBI) with approximately 100 IU/mg anticoagulant potency are actually composed by a mixture of low-anticoagulant (approximately $25 \mathrm{IU} / \mathrm{mg}$ ) and high-anticoagulant (200 IU/mg) heparin chains named $\mathrm{LABH}$ and $\mathrm{HABH}$, respectively, which can be separated through a single anion-exchange chromatography step. These bovine derivatives, as well as native $\mathrm{HBI}$ and porcine heparin ( $\mathrm{HPI}$ ), are composed by the same disaccharide units but in different proportions. $\mathrm{HABH}$ has a disaccharide composition similar to HPI while LABH contains lower proportions of N,6-disulfated and N,3,6-trisulfated $\alpha$-glucosamine units, which are important components of AT-link region of heparin. For further details on structural and pharmacological features of these heparins see Tovar et al 2019. ${ }^{18}$ 
increased proportion of $N$-sulfated but not 6-sulfated $\alpha$ glucosamine and diminished quantity of the disaccharide composed of $N, 3,6$-trisulfated $\alpha$-glucosamine linked to $\beta$ glucuronic acid (-Fig. 1), which is directly involved in the potentiation of antithrombin (AT), makes the anticoagulant activity of HBI significantly lower than that of HPI (approximately 120 vs. 180 international unit [IU]/mg, respectively). ${ }^{15-17}$ Nevertheless, our research group demonstrated that pharmaceutical HBI is actually composed of a mixture of two types of heparin chains bearing different chemical compositions and anticoagulant activities, which in turn can be separated through a single ion-exchange chromatography step (-Fig. 1), named as high-anticoagulant bovine heparin (HABH) and LABH. ${ }^{18,19}$ While HABH has chemical composition (enriched in N,6-disulfated $\alpha$-glucosamine) and anticoagulant activity (approximately $200 \mathrm{IU} / \mathrm{mg}$ ) similar to HPI, LABH has diminished potency (approximately $25 \mathrm{IU} / \mathrm{mg}$ ) due to the preponderance of disaccharides containing $\mathrm{N}$-sulfated but not 6 -sulfated $\alpha$-glucosamine (-Fig. 1). Different from HBI, HPI and pharmaceutical preparations sourced from bovine lung contain diminished amounts of low-anticoagulant heparin chains such as the component of LABH. ${ }^{18}$

Considering that LABH has anticoagulant potency markedly lower than that of the gold standard HPI and consequently reduced bleeding risk, it is a suitable candidate for prospecting novel pharmaceutical uses of UFH. ${ }^{20}$ In the present work, we evaluate the pharmacokinetics profile and bleeding potential of LABH administered subcutaneous (SC) through animal models. We also find that SC treatment with LABH delays the early Lung Lewis Carcinoma (LLC) tumor progression in mice and improves survival and brings beneficial health outcomes (reduced weight loss and incidence of complicated tumors) to the sick animals. Besides the potential for development as a new oncologic coadjutant, the establishment of basic pharmacological parameters, such as pharmacokinetics and safety, is paramount for further researches on the use of $\mathrm{LABH}$ as a therapeutic agent for treatment of other non-thromboembolic diseases.

\section{Material and Methods}

\section{Heparins}

LABH employed in the assays was prepared by fractionating a pool containing 10 batches of pharmaceutical preparations of HBI available in the Brazilian market through ion-exchange chromatography, by following the "Protocol 1" described in Tovar et al. ${ }^{18}$ Pharmaceutical HPI (Hemofol) was obtained from Cristália (Itapira, Brazil) and the LMWH enoxaparin (Clexane) from Sanofi (Singapore). The Sixth International Heparin Standard (2,145 units per vial, Lot No. 07/328) and the Third International Standard for LMWH (Lot 11/176) were obtained from the National Institute for Biological Standards and Control (NIBSC; Potters Bar, United Kingdom).

\section{Animal Experiments}

Experiments were conducted with adult (8-13 weeks age) wild type $\mathrm{C} 57 \mathrm{Bl} / 6$ mice maintained at 22 to $24^{\circ} \mathrm{C}$, artificial light cycles of 12 hours, and ad libitum feeding. The animals submitted to invasive/surgical procedures were anesthetized with $35 \mathrm{mg} / \mathrm{kg}$ ketamine and $9 \mathrm{mg} / \mathrm{kg}$ xylazine (both from Ceva Brasil; Paulínia, Brazil) administered intraperitoneally. All in vivo assays were performed in compliance with the guidelines for animal care and experimentation of our institution (Federal University of Rio de Janeiro, Brazil).

\section{In Vitro Anti-Flla and -FXa Activities}

The anticoagulant activities of the heparins were determined by measuring inhibition of thrombin (FIla) and activated factor $\mathrm{X}$ (FXa) with chromogenic assays, as previously described. ${ }^{21}$ Different concentrations of HPI, LABH, and LMWH $(0 \rightarrow 0.4 \mu-$ $\mathrm{g} / \mathrm{mL}$ ) were incubated $\left(60\right.$ seconds at $37^{\circ} \mathrm{C}$ ) with $10 \mathrm{nM}$ AT and 2 nM FIIa or FXa (Hematologic Technologies; Essex Junction, United States) or human plasma. After incubation, the anti-FIla or -FXa activities were determined by adding $100 \mu \mathrm{M}$ of chromogenic substrates S-2238 or S-2765 (Chromogenix; Molndal, Sweden), respectively, and then recording absorbance $(405 \mathrm{~nm})$ during 300 seconds in a ThermoMax Microplate Reader (American Devices; Sunnyvale, United States). Anti-FIla and -FXa potencies (IU/mg) were calculated with basis on parallel line assays performed with the Sixth International Heparin Standard (for LABH and HPI) and with the Third Standard for LMHW.

\section{Pharmacokinetic Assessments}

Plasmatic concentrations of the heparins were indirectly estimated by measuring residual anti-FXa or anti-FIIa activity, as described elsewhere. ${ }^{22}$ Briefly, animals treated with doses of 2,8 , and $20 \mathrm{mg} / \mathrm{kg}$ of $\mathrm{LMWH}, \mathrm{HPI}$, and $\mathrm{LABH}$, respectively, were administered SC. Blood samples were collected by the inferior vena cava at different times after the treatment $(0 \rightarrow 10$ hours). The anti-FXa or anti-FIla activities (IU/mL) were measured as described in the previous section by using different dilutions of the plasmas from each treatment and then calculated on the basis of the values obtained to naïve plasma spiked with known concentrations of HPI, LABH, or LMWH.

\section{Bleeding Evaluations}

Bleeding tendencies of the heparins were assessed on the basis of in vivo model described in Tovar et al. ${ }^{18}$ Briefly, animals were treated SC with different doses of $\mathrm{LABH}(8 \rightarrow 40$ $\mathrm{mg} / \mathrm{kg})$, HPI ( $1 \rightarrow 16 \mathrm{mg} / \mathrm{kg})$, LMWH $(8 \rightarrow 16 \mathrm{mg} / \mathrm{kg}$ ) and saline (control). Bleeding was quantified after 1 hour of heparin administration by collecting the blood spilled from cuts ( $1 \mathrm{~mm}$ diameter) in the tails of the animals in $1.5 \mathrm{~mL}$ distilled water. Blood was collected for 10 minutes and then in the subsequent 50 minutes and hemorrhage quantified by measuring the dissolved hemoglobin (absorbance $540 \mathrm{~nm}$ ) with a ThermoMax Microplate Reader.

\section{Lewis Lung Carcinoma Model in Mice}

LLC cells obtained from ATCC (Manassas, United States) were grown in modified Dulbecco-Eagle (DMEM) medium (Vitrocell; Campinas, Brazil), supplemented with 10\% fetal bovine serum (Invitrogen; Waltham, United States). The LLC cells 
$\left(5 \times 10^{5}\right.$ cells $\left./ 60 \mu \mathrm{L}\right)$ were inoculated SC into the dorsal region of the mice. One day after the inoculation, the animals were treated with SC injections of LABH, HPI, or LMWH $(8 \mathrm{mg} / \mathrm{kg}$ of each) or saline (control) once daily during 26 days (D27). Growing of the tumors was monitored weekly (D1, D7, D14, D21 and D28) by measuring their cranium-caudal and lateral-lateral axes. After the treatment period (D28), the animals were sacrificed and then the tumors were surgically resected and weighed.

\section{Evaluation of Health Parameters}

Different health parameters of both heath (naïve) and sick animals treated with different heparins or saline (control) were evaluated. Body weight of the animals was monitored weekly (D1, D7, D14, D21, and D28) during the treatment period. Weighing of the animals on D28 was subtracted from the tumor weights. Ectoscopic evaluations were based on the onset and severity of ulcerations and hematomas caused by the tumors or application of the heparins during or after (post-mortem) treatment period. Mortality rate of the animals submitted to different treatments was recorded daily and the cause of death was assessed by macropathological necropsy, ulceration, and/or critical health conditions were criteria to sacrifice the animal. The lungs of the animals were evaluated for the occurrence of metastasis by histological examination. A minimum of three slides of each animal, stained with hematoxylin-eosin were carefully examined.

\section{Results and Discussion}

\section{LABH Differs from HPI and LMWH on the Profile of Anticoagulant Activity}

We evaluated the in vitro anticoagulant activity of LABH based on the anti-FIIa and anti-FXa activities and compared the effect with those of HPI and LMWH. Clearly, the three types of heparins present different activity profiles. LABH had low and HPI high anticoagulant activities in both assays (approximately $25 \mathrm{IU} / \mathrm{mg}$ and approximately $180 \mathrm{IU} / \mathrm{mg}$, respectively; -Fig. 2). In contrast, LMWH (enoxaparin) showed potent anti-FXa but low anti-FIla activities (approximately $100 \mathrm{IU} / \mathrm{mg}$ and approximately $25 \mathrm{IU} / \mathrm{mg}$; - Fig. 2), as previously established elsewhere. ${ }^{22-24}$ Although its low potency had already been determined by Tovar et $\mathrm{al}^{18}{ }^{18}$ the distinct profile of LABH seen in these anticoagulant assays indicates that this heparin may also have different therapeutic effects than HPI and LMWH on non-hemostatic pathological conditions. We also found that the anti-FXa/anti-FIIa ratios of LABH in assays conducted with purified AT or human plasma (containing both AT and heparin co-factor II [HCII]) are approximately 1 (data not shown). Considering that HCII mediates anti-FIIa but not anti-FXa activity, this indicates that the contribution of $\mathrm{HCII}$ for the anticoagulant activity of LABH is similar to those previously reported in both $\mathrm{HPI}$ and $\mathrm{HBI}^{18}$

\section{LABH and HPI Administered SC Have Similar Pharmacokinetics Profile}

The following step on the way to propose a therapeutic use for LABH is to assure its absorption after SC administration and define its pharmacokinetic profile. To achieve this objective, LABH, HPI, and LMWH were administered SC to mice at doses adjusted to assure their detections in the plasma by anti-FXa assay. The low anticoagulant activity of LABH requires administration of a higher dose $(20 \mathrm{mg} / \mathrm{kg})$ to make feasible its detection in the mice plasma. LABH and HPI have similar pharmacokinetics profiles (-Fig. 3), as estimated by the time required to achieve their maximum plasma concentrations ( $\left.T_{\max }\right)$ and the half-life time $\left(T_{1 / 2}\right)$, which are markedly distinct from the profile of LMWH ( - Table 1). Clearly, we observed a similarity between the absorption of LABH and HPI but not with LMWH.

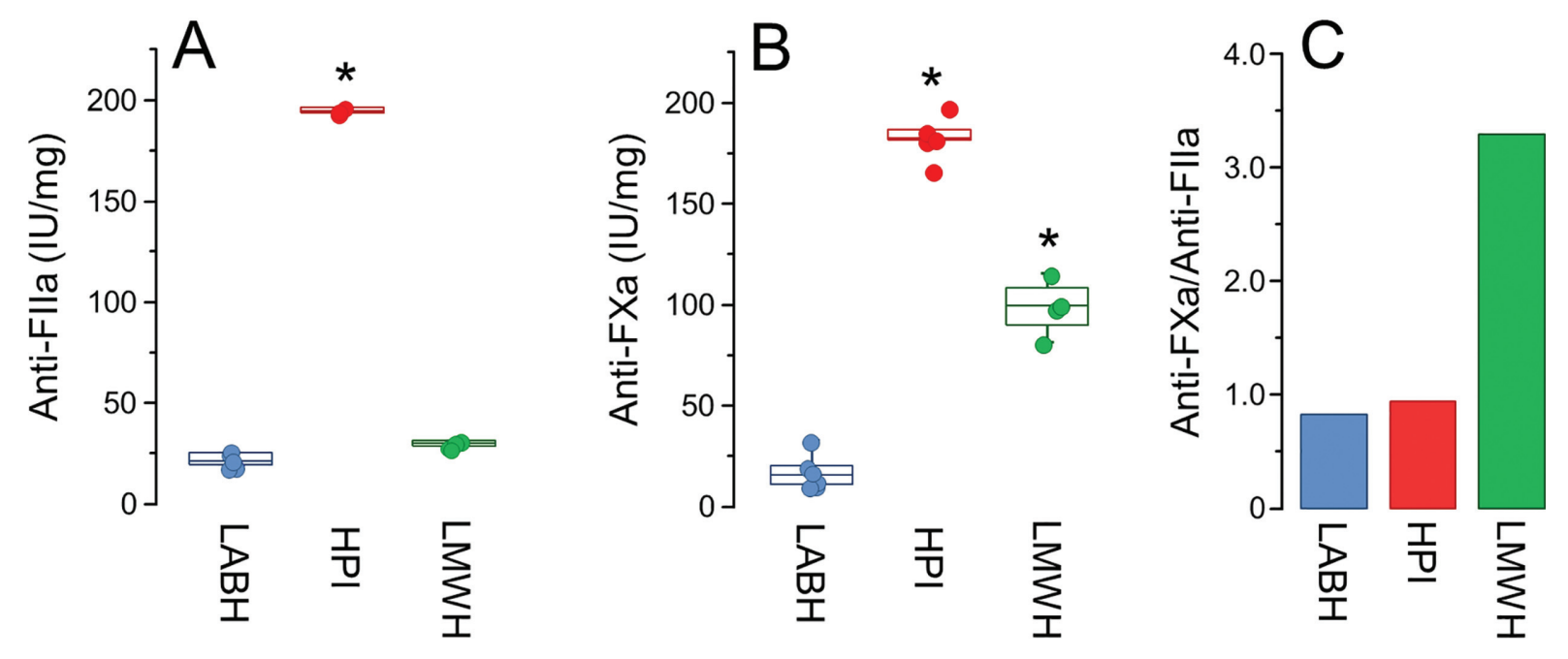

Fig. 2 Anti-Flla (A), anti-FXa (B) activities and anti-FXa/anti-Flla ratios (C) of LABH, HPI, and the LMWH enoxaparin. Results expressed as IU/mg were calculated with basis on parallel line assays performed with the $6^{\text {th }}$ International Heparin Standard for LABH and HPI and with the $3^{\text {rd }}$ Standard for LMWH. ${ }^{*}$ indicates $p<0.05$ in relation to LABH (ANOVA with Bonferroni post hoc test). 

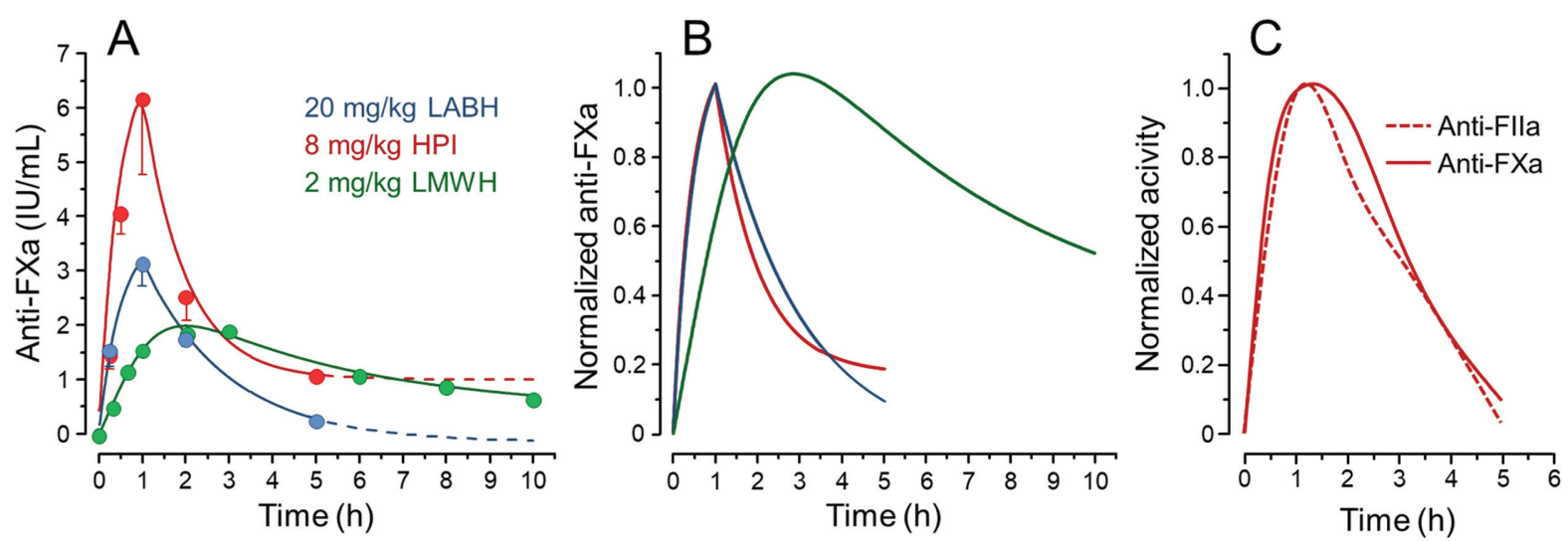

Fig. 3 Pharmacokinetics profiles of HABH, HPI, and LMWH. Doses of 2, 8, and $20 \mathrm{mg} / \mathrm{kg}$ body weight of LMWH, HPI, and LABH, respectively, were SC administered to mice. Blood samples were collected by the inferior vena cava at different periods of time and heparin concentrations in the plasma were determined by the anti-FXa assay. Panel (A) shows the effective results and panel (B) the data for each heparin normalized to a maximum value of 1 based on plasma anti-FXa activities and the panel (C) the comparison of normalized profiles of HPI monitored by anti-FXa (solid red line) or anti-Flla (dashed red line) activities.

Table 1 Pharmacokinetic parameters $T_{\max }$ (time to reach maximum plasma concentration), $T_{1 / 2}$ (elimination half-life), $A U C_{0-10 h}$ (area under the curve) and $C_{\max }$ (maximum plasma concentration) and molecular masses $\left(M_{w}\right.$ ) of LABH, HPI, and LMWH

\begin{tabular}{|l|l|l|l|l|l|}
\hline & $T_{\max }(\mathrm{h})$ & $T_{1 / 2}(\mathrm{~h})$ & $\mathrm{AUC}_{0-10 \mathrm{~h}}(\mu \mathrm{g} / \mathrm{mL})^{\mathrm{a}}$ & $C_{\max }(\mu \mathrm{g} / \mathrm{mL})^{\mathrm{a}}$ & $M_{w}(\mathrm{Da})^{\mathrm{b}}$ \\
\hline LABH & 1 & $0.84 \pm 0.3$ & 42.0 & 16.5 & approximately 13,500 \\
\hline HPI & 1 & $1.52 \pm 0.8$ & 25.5 & 8.5 & approximately 17,000 \\
\hline LMWH & 2 & $4.89 \pm 0.2$ & 126.0 & 19.9 & approximately 4,500 \\
\hline
\end{tabular}

Abbreviations: LABH, low-anticoagulant bovine heparin; LMWH, low-molecular weight heparin.

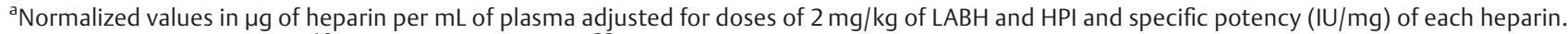
${ }^{\mathrm{b}}$ Data from Tovar et al $2019^{18}$ and Oliveira et al $2015 .^{22}$

When the maximum plasma concentrations $\left(C_{\max }\right)$ and also the area under the curve (AUC) were normalized to a similar dose and to their specific anti-FXa potencies, we observed that LABH is better absorbed than HPI but poorer than LMWH ( - Table 1). Although differing in the proportion of some disaccharide building blocks, the improved absorption of LABH in comparison to HPI certainly relates to its smaller molecular weight (13.5 and $17 \mathrm{kDa}$, respectively), which confirms the inverse correlation between SC suitability and molecular weights of the heparins. ${ }^{25}$ We also observed that the pharmacokinetics profiles of HPI administered SC by monitoring anti-FXa or anti-FIla activities are similar (-Fig. 3C). Although there is little information on the pharmacokinetics of UFHs after SC administration, our results are in line with the literature reporting that high-molecular-mass heparins have poor SC pharmacokinetics and are eliminated by both renal filtration and endothelium cells capitation while LMWHs are better absorbed and removed from plasma mostly by renal route. ${ }^{26}$

\section{LABH-Administered SC Does Not Provoke Bleeding}

Next, we evaluated the bleeding effect of LABH after SC administration. Bleeding is the major adverse effect of heparin, especially in the case of UFHs. ${ }^{27,28}$ It is an obstacle for the use of UFHs in non-thromboembolic diseases, especially because such uses often require elevated doses and long periods of administration. ${ }^{29}$ We evaluated bleeding measuring the blood spilled from cuts in tails of mice 1 hour after SC administration of LABH, HPI, and LMWH. Blood was collected for an initial period of 10 minutes and then in the subsequent 50 minutes (-Fig. 4).

In the initial 10 minutes of blood collection, none of the heparins provoked blood losses significantly higher than those of the animals treated with saline (control) (-Fig. 4A). Possibly, the mechanisms of primary hemostasis (e.g., vasoconstriction, change in vascular permeability and platelet adhesion) are able to assuage the initial bleeding, even in the animals heparinized with HPI. ${ }^{30,31}$ In the subsequent 50 minutes, doses of HPI and LMWH above $8 \mathrm{mg} / \mathrm{kg}$ resulted in blood losses significantly higher than that measured in control animals ( $\boldsymbol{- F i g . ~ 4 B )}$. On the other hand, SC administration of LABH did not increase bleeding, except for a modest effect in the animals treated with a very high dose $(40 \mathrm{mg} / \mathrm{kg})$.

Tovar et al showed that different from HPI, HBI, and the bovine derivative HABH, LABH did not provoke bleeding even by intravenous (IV) administration. ${ }^{18}$ Such a lack of hemorrhage effects directly correlates with the low anticoagulant potency of LABH (approximately $25 \mathrm{IU} / \mathrm{mg}$ ). Although LMWHs are certainly safer than non-modified UFHs, ${ }^{32,33}$ we observed that even high SC doses of LABH provoked less bleeding than enoxaparin in the animals, this is possibly 


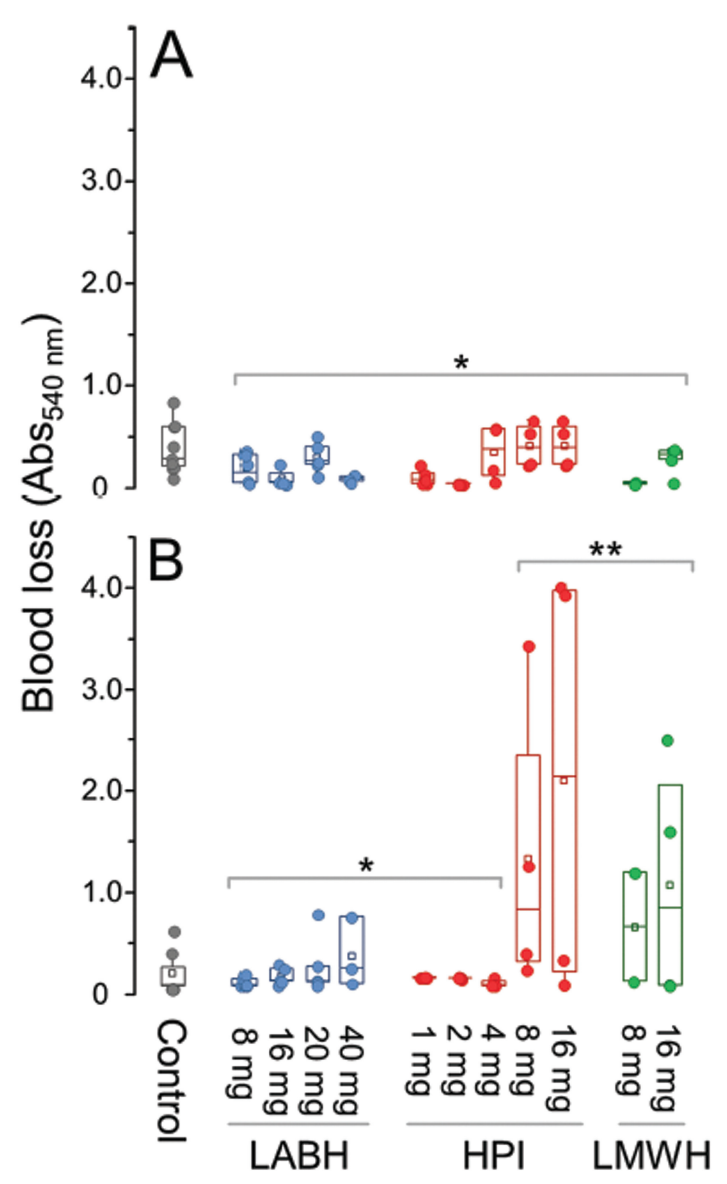

Fig. 4 Bleeding evaluations of the three types of heparins. Different doses of $\mathrm{LABH}, \mathrm{HPI}, \mathrm{LMWH}$, and saline (control) were administered SC to mice. Bleeding was quantified by collecting the blood spilled from cuts in the tail of the animals in distilled water for an initial period of 10 minutes (A) and in the subsequent 50 minutes (B). The dissolved hemoglobin was determined by absorbance at $540 \mathrm{~nm} .{ }^{*}$ indicates $p>0.05$ and ${ }^{* *} p<0.05$ in relation to the control (ANOVA with Bonferroni post hoc test).

due to the improved SC absorption and high anti-FXa potency (approximately $100 \mathrm{IU} / \mathrm{mg}$ ) of this LMWH. In conclusion, LABH is devoid of bleeding effect by both SC and IV administration and thus do not pose the worse adverse effect hindering the therapeutic use of UFHs in non-thromboembolic diseases.

\section{Effect of LABH on LLC Tumor Progression}

Anticancer effect is one of the most relevant non-hemostatic pharmacological activities reported for heparins. ${ }^{34-36} \mathrm{We}$ assessed this effect of LABH in comparison with those of HPI and LMWH using an experimental model of cancer based on tumor formation by SC inoculation of LLC cells in mice. ${ }^{37}$ The animals received daily SC doses $(8 \mathrm{mg} / \mathrm{kg})$ of the three heparins. Measurement of the tumor size by examination of the animals showed that the three heparins delayed tumor progression, which is more evident up to the $14^{\text {th }}$ day and became less expressive on the examinations at the $21^{\text {st }}$ and especially at the $28^{\text {th }}$ days ( $\boldsymbol{\sim}$ Fig. $\mathbf{5 A}$ ). Tumor was detected on the $14^{\text {th }}$ day in nine animals among 15 receiving saline and only in four among 10 treated with LABH, all with a small size $\left(<0.5 \mathrm{~cm}^{2}\right)$. None of the seven animals treated with LMWH had tumor and just one among the same number of animals was treated with HPI (shadowed area in - Fig. 5A).

On the $28^{\text {th }}$ day after tumor cells inoculation, the animals were sacrificed and their tumors resected, examined, and weighted. Again, we observed that the heparins delayed tumor progression, which was less evident in the case of LMWH (-Fig. 5B, C). Animals treated with LABH and HPI showed a decrease in tumor size, but with no statistical significance, in comparison with the control animals. We did not identify tumor metastasis on lungs of all animals' groups after careful histological examination (not shown).

Subsequently we attempted to examine the balance between the beneficial action of the three heparins on the tumor progression and possible adverse effects of the drugs. The relative survival curves ( $\mathbf{- F i g . ~ 6 A ) ~ s h o w e d ~ t h a t ~ a n i m a l s ~}$ inoculated with LLC cells and treated with HPI or saline had a high mortality rate, most of them were related to ulcerations. Some animals treated with HPI that died prematurely showed extensive post-mortem dorsal hematoma at the injection site, in addition to pulmonary hemorrhages, suggesting that bleeding is the major cause of death or at least contributed to that. These findings did not occur in other groups of mice. The animals treated with LABH and LMWH showed a notable decrease in mortality compared with the animals treated with saline or HPI.

Another approach to examine the beneficial effects of the heparins during tumor progression was based on the ponderal loss/gain of body weight in the course of 28 days treatment. Mice inoculated with LLC cells and treated with LABH showed an average body weight increase of approximately $11 \%$, similar to the naive group (approximately $14 \%$ ) (-Fig. 6B). Animals treated with saline (control) had a decrease of $9 \%$ in body weight while HPI and LMWH had more modest effect on the increase of the mice body weight (approximately 3 and 6\%, respectively). We also evaluated the incidence of complicated tumors in the animals based on ectoscopic examination. The three types of heparins had favorable effects preventing tumor ulceration and hematomas but LABH and LMWH showed more pronounced benefices (-Fig. 6C).

Although most of the studies on the anticancer properties of heparins are focused on their P-selectin-mediated hematogenous anti-metastatic activities, both UFHs and LMWHs have also proven to be effective for other cancer therapeutic targets, such as upregulation of E-cadherin and inhibition of HGF, heparinase, and galectin-3..$^{38-42}$ UFH inhibits proliferation of tumor cells by modulating the proto-oncogenes c-Myc and c-Fos, which downregulates the phosphorylation of MAPK part of the signaling cascade of protein kinase C, thus hindering the growth of both primary and metastatic tumors. ${ }^{43}$ Another noteworthy anticancer effect of heparins is the release of the natural anticoagulant TFPI (tissue factor pathway inhibitor) from the endothelial cells. TFPI has been shown to inhibit hypercoagulability and angiogenesis resulting from the overexpression of tissue factor by tumor cells. ${ }^{44}$ Besides these hemostatic effects, TFPI, especially TFPI-2 synthesized by vascular cells, have also been demonstrated 
to exert anti-metastatic activity by downregulating effectors involved in the degradation of the extracellular matrix during extravasation or intravasation, such as heparanase and matrix metalloproteinase- $1 .{ }^{45,46}$

Other heparin/heparinoid derivatives devoid of anticoagulant activity have already exhibited anticancer effects. A heparan sulfate hexasaccharide has proven to inhibit cancer stem cells renewal and induces apoptosis of three types of tumor cells. ${ }^{47}$ Another study tested a low-anticoagulant heparin on patients with myeloid leukemia, aiming the survival of the leukemic stem cells in the marrow bone, which resulted in increased remission/recovery rates and no
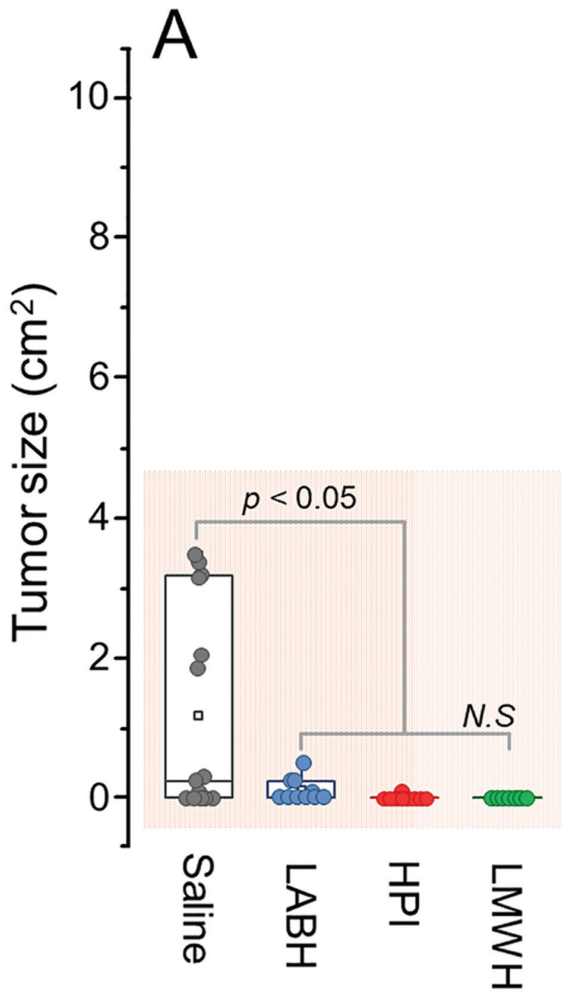

Day $1 \rightarrow 14$

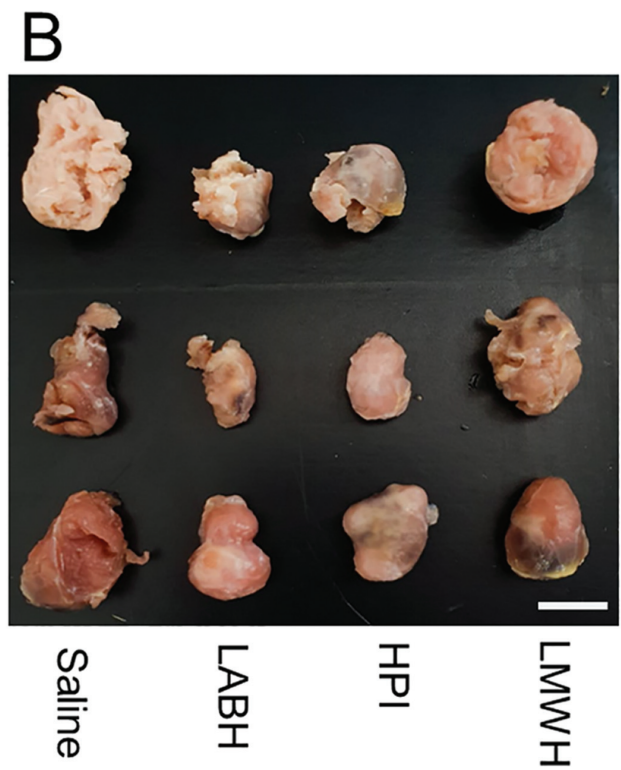

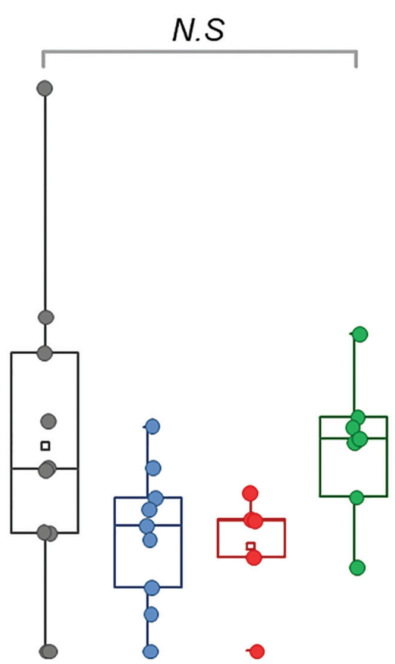

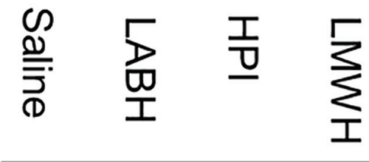

Day 21
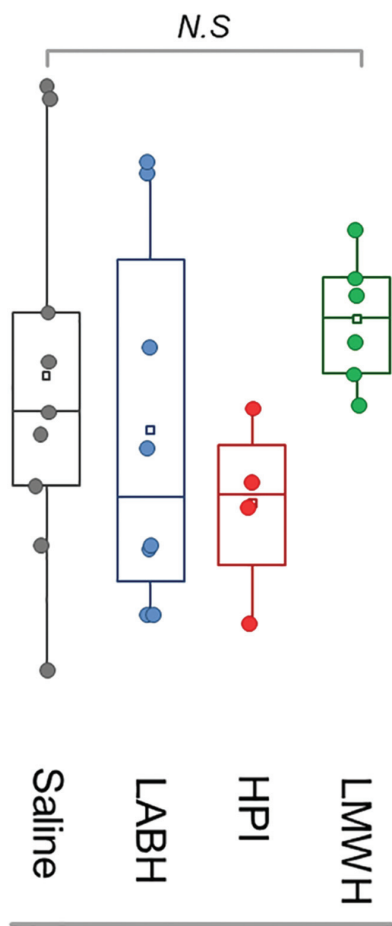

Day 28

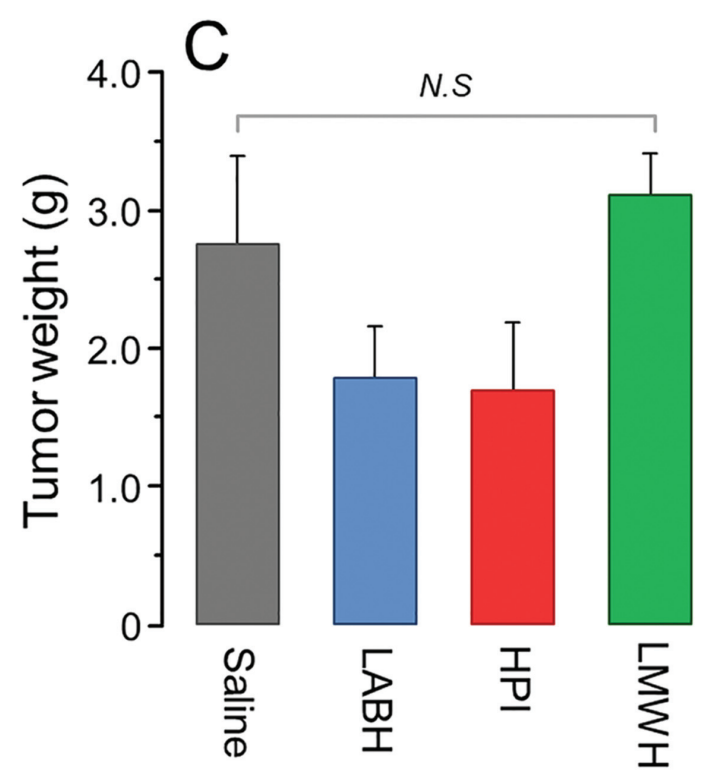

Fig. 5 Progression of LLC tumors in mice treated with LABH, HPI, LMWH, and saline (control). LCC cells ( $5 \times 10^{5}$ cells in $60 \mu \mathrm{L}$ ) were inoculated SC into the dorsal region of mice. (A) One day after inoculation, the mice received daily SC injections of the different heparins for 28 days. Growing of the tumors was monitored weekly measuring their cranium-caudal and lateral-lateral axes. On the $28^{\text {th }}$ day the animals were sacrificed and then the tumors were resected (B) and weighed (C). N.S., non-significant ( $p>0.05$; ANOVA with Bonferroni post hoc test). 

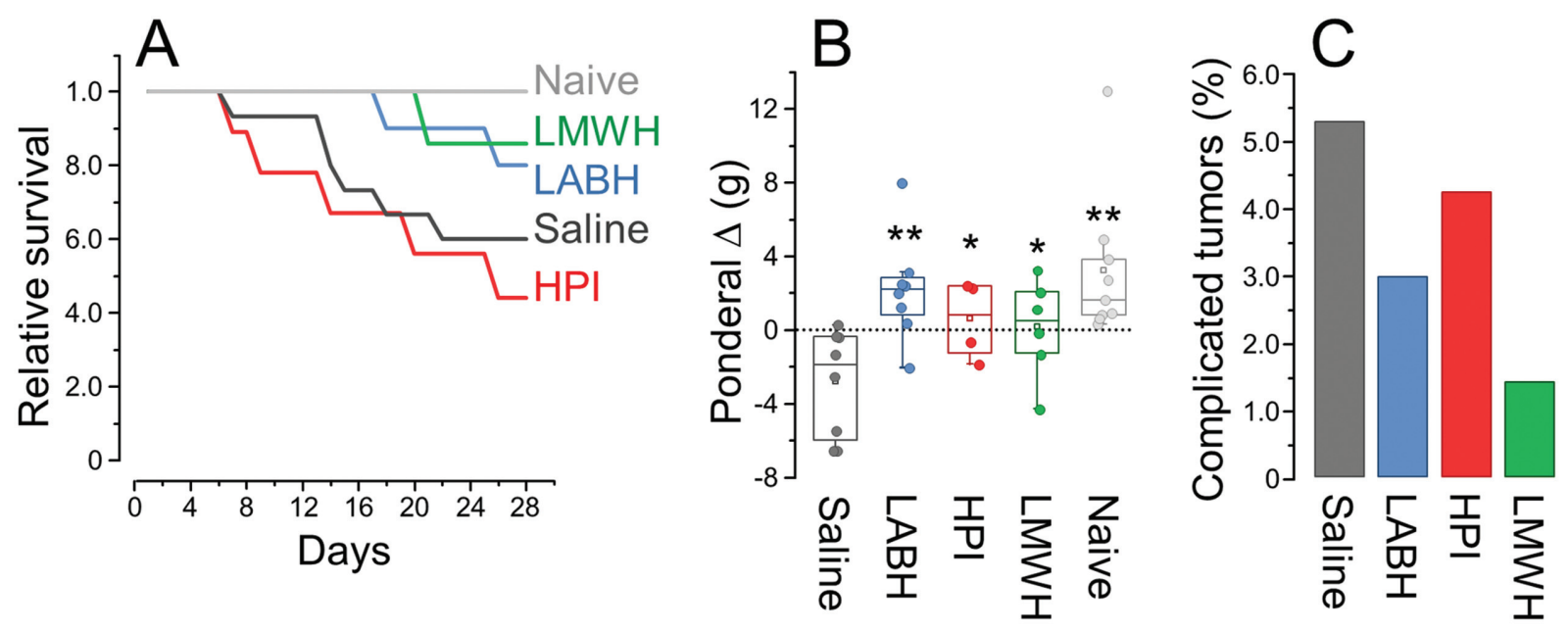

Fig. 6 Relative survival (A), ponderal weight variation (B), and percentage of complicated tumors (C) of mice inoculated with LLC cells treated with the different heparins and saline (control) and naïve animals. ${ }^{*}$ indicates $p>0.05$ and ${ }^{* *} p<0.05$ in relation to the control (ANOVA with Bonferroni post hoc test).

adverse events. ${ }^{48}$ The sulfated non-anticoagulant heparin (S$\mathrm{NACH}$ ) increases threefold TFPI-2 levels and suppresses pancreatic tumor growth and metastasis in animal models. ${ }^{49,50}$ Notwithstanding we have not demonstrated a specific mechanism of action, the delay in the early LLC tumor progression, improved survival and health (reduced weight loss and incidence of complicated tumors) promoted by SC administration of doses deprived of bleeding risk of the lowanticoagulant LABH in the mice must not relates to survival/ viability of cancer stem cells, considering that it did not affect the final size of the tumors, but to a possible raise in the plasmatic levels of TFPI.

\section{Conclusion}

Several clinical trials have shown that both UFH and LMWHs might bring beneficial clinical outcomes to patients with different solid tumors; nevertheless, the elevated risk of hemorrhage incidents jeopardizes their uses. ${ }^{51}$ The main objective of this study was to evaluate the effect of a heparin with low-anticoagulant activity (named as LABH), a derivative from pharmaceutical preparations of HBI, on tumor progression in mice. The effect of this derivative was compared with that of the gold standards HPI and enoxaparin (LMWH). The three types of heparins were SC administered to mice at the same dose in mass and not as anticoagulant potency (IU) since the effect we tested is not related to the action of the heparins on coagulation.

The administration of HPI delayed tumor progression but in parallel it had a toxic effect in the animals, as indicated by the increased mortality, some due to bleeding. In contrast, LABH had a similar effect in hindering tumor progression, but without such deleterious effects. These observations show that LABH has a wider therapeutic window than HPI, with beneficial pharmacological action and diminished adverse effects. Considering that UFHs are commonly administered IV, another challenge we overcome here was to ensure that LABH is satisfactorily absorbed after SC administration, which is an essential route for outpatient use over long periods of time.

Finally, our study is an example of combining the detailed structural analysis of heparin preparations with testing their pharmacological effects on both in vitro assays and specific animal experimental models. This is an approach to obtain more accurate information on the structure versus specific biological effects, which may lead to the development of new heparins with practical use in medicine.

\section{What Is Known about This Topic?}

- Heparin has several pharmacological properties other than anticoagulant activity, especially for use as new oncogenic agent.

- However, the high anticoagulant potency of heparin makes the doses necessary to exert non-hemostatic effects unsafe due to an elevated bleeding risk.

- Development of new heparins with decreased anticoagulant activity could make the therapeutic use for treating non-thromboembolic diseases safer.

\section{What Does This Paper Add?}

- We demonstrate that a low-anticoagulant bovine heparin derivative is suitable for subcutaneous administration required for outpatient/long-term treatments.

- Even high subcutaneous doses of this derivative do not provoke bleeding.

- This derivative was able to delay the early progression of Lewis lung carcinoma, improve survival, and bring health benefits with minimal adverse effects.

- Such a low-anticoagulant heparin is a promising candidate for development as a new therapeutic agent for treatment of non-thromboembolic diseases. 


\section{Authors' Contributions}

A.M.F.T., R.P.S., and P.A.S.M. conceived the research and experimental design; A.M.F.T., R.P.S, A.A.P., M.R.O., N.V.C., S.N.M.C.G.O., J.N.F, and A.H.C conducted the research; A.M. F.T., R.P.S., S.N.M.C.G.O., and E.V. analyzed the data, and R. P.S., A.M.F.T., E.V., and P.A.S.M. wrote the paper.

\section{Funding}

This work was supported by the grants $315847 / 2020-4$ from Conselho Nacional de Desenvolvimento Cientifico e Tecnológico (CNPq) and E26/290.078/2017 from Fundação de Amparo à Pesquisa do Estado do Rio de Janeiro (FAPERJ).

\section{Conflict of Interest}

None declared.

\section{Acknowledgments}

The authors would like to thanks Juliana M. Motta and Carlos R. Fernandes for assisting in LLC cell culture.

\section{References}

1 Torri G, Naggi A. Heparin centenary-an ever-young life-saving drug. Int J Cardiol 2016;212(Suppl 1):S1-S4

2 Gunaratne R, Kumar S, Frederiksen JW, et al. Combination of aptamer and drug for reversible anticoagulation in cardiopulmonary bypass. Nat Biotechnol 2018;36(07):606-613

3 Cassinelli G, Naggi A. Old and new applications of non-anticoagulant heparin. Int J Cardiol 2016;212(Suppl 1):S14-S21

4 Hippensteel JA, LaRiviere WB, Colbert JF, Langouët-Astrié CJ, Schmidt EP. Heparin as a therapy for COVID-19: current evidence and future possibilities. Am J Physiol Lung Cell Mol Physiol 2020; 319(02):L211-L217

5 Chen D. Heparin beyond anti-coagulation. Curr Res Transl Med 2021;69(04):103300

6 Atallah J, Khachfe HH, Berro J, Assi HI. The use of heparin and heparin-like molecules in cancer treatment: a review. Cancer Treat Res Commun 2020;24:100192

7 Van Haren RM, Rajaram R, Correa AM, et al. Preoperative heparin for lung cancer resection increases risk of reoperation for bleeding. Semin Thorac Cardiovasc Surg 2020;32(02):337-343

8 Yamaura G, Ito T, Miyaji Y, et al. Therapeutic efficacy of heparin and direct factor Xa inhibitors in cancer-associated cryptogenic ischemic stroke with venous thromboembolism. Thromb Res 2021;206:99-103

9 Mousa SA. Heparin, low molecular weight heparin, and derivatives in thrombosis, angiogenesis, and inflammation: emerging links. Semin Thromb Hemost 2007;33(05):524-533

10 Vilanova E, Tovar AMF, Mourão PAS. Imminent risk of a global shortage of heparin caused by the African Swine Fever afflicting the Chinese pig herd. J Thromb Haemost 2019;17(02):254-256

11 van der Meer JY, Kellenbach E, van den Bos LJ. From farm to pharma: an overview of industrial heparin manufacturing methods. Molecules 2017;22(06):E1025

12 Al-Hakim A. General Considerations for Diversifying Heparin Drug Products by Improving the Current Heparin Manufacturing Process and Reintroducing Bovine Sourced Heparin to the US Market. Clin Appl Thromb Hemost 2021; 10760296211052293

13 Gomes WJ, Leal JC, Braile DM, et al. A Brazilian perspective for the use of bovine heparin in open heart surgery. Int J Cardiol 2016; 223:611-612
14 Vilanova E, Vairo BC, Oliveira SMCG, et al. Heparins sourced from bovine and porcine mucosa gain exclusive monographs in the Brazilian Pharmacopeia. Front Med (Lausanne) 2019;6:16

15 Tovar AMF, Capillé NV, Santos GR, et al. Heparin from bovine intestinal mucosa: glycans with multiple sulfation patterns and anticoagulant effects. Thromb Haemost 2012;107(05): 903-915

16 Tovar AMF, Teixeira LA, Rembold SM, Leite M Jr, Lugon JR, Mourão PA. Bovine and porcine heparins: different drugs with similar effects on human haemodialysis. BMC Res Notes 2013;6:230

17 Tovar AMF, Santos GR, Capillé NV, et al. Structural and haemostatic features of pharmaceutical heparins from different animal sources: challenges to define thresholds separating distinct drugs. Sci Rep 2016;6:35619

18 Tovar AMF, Vairo BC, Oliveira SMCG, et al. Converting the distinct heparins sourced from bovine or porcine mucosa into a single anticoagulant drug. Thromb Haemost 2019;119(04): 618-632

19 Mourão PAS, Medeiros LN, Vilanova E, Aquino RS inventors; Heptech Ltda., assignee. Safe Bovine Heparin, Preparation Method and Application. WIPO Patent No. 2020/163926 A1. August 20, 2020

20 Silva CFS, Motta JM, Teixeira FCOB, et al. Non-anticoagulant heparan sulfate from the ascidian Phallusia nigra prevents colon carcinoma metastasis in mice by disrupting platelet-tumor cell interaction. Cancers (Basel) 2020;12(06):1353

21 Glauser BF, Santos GRC, Silva JD, et al. Chemical and pharmacological aspects of neutralization of heparins from different animal sources by protamine. J Thromb Haemost 2018;16(09): 1789-1799

22 Oliveira SN, Santos GR, Glauser BF, et al. Structural and functional analyses of biosimilar enoxaparins available in Brazil. Thromb Haemost 2015;113(01):53-65

23 Glauser BF, Vairo BC, Oliveira CP, Cinelli LP, Pereira MS, Mourão PA. Generic versions of enoxaparin available for clinical use in Brazil are similar to the original drug. J Thromb Haemost 2011;9(07): $1419-1422$

24 Vilanova E, Glauser BF, Oliveira SM, Tovar AMF, Mourão PAS. Update on Brazilian biosimilar enoxaparins. Expert Rev Hematol 2016;9(11):1015-1021

25 Kandrotas RJ. Heparin pharmacokinetics and pharmacodynamics. Clin Pharmacokinet 1992;22(05):359-374

26 Fareed J, Hoppensteadt D, Walenga J, et al. Pharmacodynamic and pharmacokinetic properties of enoxaparin : implications for clinical practice. Clin Pharmacokinet 2003;42(12):1043-1057

27 Krishnaswamy A, Lincoff AM, Cannon CP. Bleeding complications of unfractionated heparin. Expert Opin Drug Saf 2011;10(01): $77-84$

28 Piran S, Schulman S. Treatment of bleeding complications in patients on anticoagulant therapy. Blood 2019;133(05): 425-435

29 Castelli R, Porro F, Tarsia P. The heparins and cancer: review of clinical trials and biological properties. Vasc Med 2004;9(03): 205-213

30 Riddel JP Jr, Aouizerat BE, Miaskowski C, Lillicrap DP. Theories of blood coagulation. J Pediatr Oncol Nurs 2007;24(03):123-131

31 Lippi G, Favaloro EJ, Franchini M, Guidi GC. Milestones and perspectives in coagulation and hemostasis. Semin Thromb Hemost 2009;35(01):9-22

32 Costantino G, Ceriani E, Rusconi AM, et al. Bleeding risk during treatment of acute thrombotic events with subcutaneous LMWH compared to intravenous unfractionated heparin; a systematic review. PLoS One 2012;7(09):e44553

33 Lazrak HH, René É, Elftouh N, Leblanc M, Lafrance JP. Safety of lowmolecular-weight heparin compared to unfractionated heparin in hemodialysis: a systematic review and meta-analysis. BMC Nephrol 2017;18(01):187 
34 Mohan CD, Hari S, Preetham HD, et al. Targeting heparanase in cancer: inhibition by synthetic, chemically modified, and natural compounds. iScience 2019;15:360-390

35 Andrgie AT, Birhan YS, Mekonnen TW, et al. Redox-responsive heparin-chlorambucil conjugate polymeric prodrug for improved anti-tumor activity. Polymers (Basel) 2019;12(01):43

36 Sarantis P, Bokas A, Papadimitropoulou A, et al. Combinatorial treatment of tinzaparin and chemotherapy can induce a significant antitumor effect in pancreatic cancer. Int J Mol Sci 2021;22(13):7053

37 Choi JG, Kim JM, Kang DW, et al. Inoculation of Lewis lung carcinoma cells enhances formalin-induced pain behavior and spinal Fos expression in mice. J Vet Sci 2017;18(03):267-272

38 Borsig L. Antimetastatic activities of heparins and modified heparins. Experimental evidence. Thromb Res 2010;125(Suppl2): S66-S71

39 Chen Y, Peng J, Han M, et al. A low-molecular-weight heparincoated doxorubicin-liposome for the prevention of melanoma metastasis. J Drug Target 2015;23(04):335-346

40 Vijaya Kumar A, Salem Gassar E, Spillmann D, et al. HS3ST2 modulates breast cancer cell invasiveness via MAP kinase- and Tcf4 (Tcf712)-dependent regulation of protease and cadherin expression. Int J Cancer 2014;135(11):2579-2592

41 Weissmann M, Arvatz G, Horowitz N, et al. Heparanase-neutralizing antibodies attenuate lymphoma tumor growth and metastasis. Proc Natl Acad Sci U S A 2016;113(03):704-709

42 Fortuna-Costa A, Gomes AM, Kozlowski EO, Stelling MP, Pavão MS. Extracellular galectin-3 in tumor progression and metastasis. Front Oncol 2014;4:138
43 Uzun Y, Akdogan E, Ozdemir F, Ovali E. The effects of heparin on DLD-1 colon cancer cell line. Bratisl Lek Listy 2009;110(01):3-6

44 Mousa SA. Anti-thrombotics in thrombosis and cancer. Future Oncol 2005;1(03):395-403

45 Nevo N, Ghanem S, Crispel Y, et al. Heparanase level in the microcirculation as a possible modulator of the metastatic process. Am J Pathol 2019;189(08):1654-1663

46 Liu J, Xie J, Huang Y, Xie J, Yan X. TFPI-2 inhibits the invasion and metastasis of bladder cancer cells. Prog Urol 2021;31(02): 71-77

47 Patel NJ, Sharon C, Baranwal S, Boothello RS, Desai UR, Patel BB. Heparan sulfate hexasaccharide selectively inhibits cancer stem cells self-renewal by activating p38 MAP kinase. Oncotarget 2016; 7(51):84608-84622

48 Kovacsovics TJ, Mims A, Salama ME, et al. Combination of the low anticoagulant heparin CX-01 with chemotherapy for the treatment of acute myeloid leukemia. Blood Adv 2018;2(04):381-389

49 Darwish NHE, Godugu K, Mousa SA. Sulfated non-anticoagulant low molecular weight heparin in the prevention of cancer and non-cancer associated thrombosis without compromising hemostasis. Thromb Res 2021;200:109-114

50 Sudha T, Yalcin M, Lin HY, et al. Suppression of pancreatic cancer by sulfated non-anticoagulant low molecular weight heparin. Cancer Lett 2014;350(1-2):25-33

51 Kuderer NM, Khorana AA, Lyman GH, Francis CW. A meta-analysis and systematic review of the efficacy and safety of anticoagulants as cancer treatment: impact on survival and bleeding complications. Cancer 2007;110(05):1149-1161 